\title{
Scanning Procedure of Female Torso Using Low-Cost Hand-Held Sense ${ }^{T M}$ 3D Scanner
}

\author{
Michaela HASSMANN ${ }^{* 1}$, Seraphina STÖGER ${ }^{1}$, Jacqueline DASTL ${ }^{2}$, Wolfgang KRACH ${ }^{2}$ \\ ${ }^{1}$ University of Vienna, Vienna, Austria; \\ ${ }^{2} \mathrm{CAE}$ Simulation \& Solutions $\mathrm{GmbH}$, Vienna, Austria
}

DOI: $10.15221 / 18.074 \quad$ http://dx.doi.org/10.15221/18.074

\begin{abstract}
3D scanning technology is widely used in medical and clothing applications as well as research projects. This paper presents our experiences with a low-cost hand-held $1^{\text {st }}$ generation Sense ${ }^{\mathrm{TM}}$ 3D scanner (3D Systems Inc., Rock Hill, SC, USA) including free software Sense (V2.2) [1]. The shape of the female torso, including breast tissue volume, was determined using 3D surface scans. Female upper body area implies special requirements for positioning of participant and handling of scanner to gain good scan quality for reliable surface geometry. Lighting, settings and options, advantages and drawbacks of this scanner, including dimensional tolerance and repeatability measurements using mannequin and human body are addressed. Data processing and anonymization issues in the included free software are shown. Dimensional accuracy has to be proven first before using the Sense ${ }^{\mathrm{TM}} 3 \mathrm{D}$ scanner. For this purpose, a rigid plastic mannequin with markups, so-called fiducial points, was used. The distance from incisura jugularis to umbilicus height and nipple distance were measured using manual anthropometry compasses and virtual measurement from the 3D scan. The deviation was $0.1 \%$ and $-0.03 \%$, respectively. Repeatability of measurements was determined calculating average error parameter from 5 scans in standing and supine position (lying on the back) using the alignment procedure in MeshLab ( $v 1.3 .4 \mathrm{BETA}$ ) [2]. Mean average error was $0.26 \mathrm{~mm}$ for both standing and supine position using either 4 or 19 pairs of points for alignment. Keeping in mind that the scanner is optimized for human skin and not for plastic surface, which sometimes causes reflections, this deviation can be judged very low. In addition, repeatability measurements were carried out on 3 pilot study participants. Mean average error for all participants and positions was $1.33 \mathrm{~mm}$. Compared to the element size of maximum $3 \mathrm{~mm}$ this error is acceptable. Hence, the low-cost Sense ${ }^{\mathrm{TM}}$ scanner can be used in research projects dealing with human body geometric measurements.
\end{abstract}

Keywords: dimensional accuracy, repeatability, handling and usability, data processing

\section{Introduction}

3D scanning technology has evolved from large to handy and from costly to low-cost $[3,4]$. This has widened its use in medical and clothing applications as well as research projects. In contrast to stationary 3D scanners, for example the 3dMD photogrammetric system [5], non-stationary 3D scanners allow their application in varying surroundings, such as small laboratories, medical practice and clinics as well as fashion shops. Scientific and commercial non-stationary 3D scanning solutions range from portable multi-camera systems [6], Microsoft Kinect ${ }^{\mathrm{TM}}$ sensor [7] to hand-held 3D scanners. Prices for hand-held 3D scanners for full-body application range from about $€ 200$ to 500 , e.g. 3D Scanner $1.0 \mathrm{~A}$ [8] or Sense ${ }^{\mathrm{TM}}$ [1] up to high-end products for $€ 5.000$ to 20.000 and more, e.g. EinScan-Pro+ [9] or Artec Eva [10]. The technology behind is either structured light (white light LED), laser triangulation (usually class I laser for human purpose) or infrared light (IR).

Choppin et al. [4] mention technological developments in 3D scanning systems with special regard to the female breast region. The Microsoft Kinect ${ }^{\mathrm{TM}}$ was found to be a very simple and cheap device for the evaluation of surgery outcome [11] with comparable accuracy to high resolution passive stereophotogrammetry systems such as in [12]. Oliveira et al. [13] list a number of studies using different 3D scanning systems for clinical breast evaluation. Koban, Schenck and Giunta [3] compare three different mobile hand-held scanning systems (Sense ${ }^{\mathrm{TM}}$, iSense ${ }^{\mathrm{TM}}$, Artec Eva) for a similar purpose, whereas the comparison of scan quality and accuracy is shown only for leg area scans. The Sense ${ }^{\mathrm{TM}}$ scanner was found to be limited in more complex surfaces such as the inframammary fold, for which it was inferior to the more costly Artec Eva scanner.

\footnotetext{
*michaela.hassmann@univie.ac.at; +43- 1- 4277- 48883; http://zsu-schmelz.univie.ac.at/en/
} 
The specific challenge in scanning and evaluation of the female breast region is the limited visibility of crucial areas in standing position, such as the lateral region close to the axilla or the inframammary fold (IMF). While the visibility of the lateral region of the breast is improved with holding the arms up, either horizontal or above the head [13], breast shape changes significantly due to skin movement and muscle tone. IMF is typically occluded from the upright view in women with large, ptotic breasts $[5,14]$. Visibility of the inframammary fold is considered optimal when the participant lies prone across two tables with a gap between the tables where the breasts can hang freely [14]. This position might be quite uncomfortable both for participants with large breasts and for the researcher who has to move the 3D scanner below the table. When lying on the back (supine), visibility of IMF is given in any grade of ptosis [15], while the grade of ptosis cannot be defined. Both prone and supine position lead to better visibility of IMF with the drawback of differing breast shape, which changes between supine, upright and prone position under gravity loading due to non-rigidity of human tissues [15]. This can be accepted if the purpose of 3D scanning is the definition of breast surface or inframammary fold only.

The necessity of female breast shape and volume assessment is given in several fields. Clothing industry aims at mass customization from hundreds to thousands of scans, or at made-to-measure [16]. The prevalent - and most often only - scanning position in clothing applications is the standing position. In aesthetic and reconstructive surgery, inframammary fold, breast volume and symmetry are determined pre and post surgery [5,11]. Different scanning positions are used due to the fact that the patient is lying supine during surgery, while the result should be optimal in upright view [15]. In biomechanics, in addition to the breast shape, the material properties of the female breast are of interest. Young's modulus for linear elastic or hyperelastic material models is calculated from the breast shape change between different positions [17].

In the recently finished research project "Sports bra optimization by Finite Element simulation of interaction between textile and female breast tissue (FEM-Sports-Bra)", breast tissue volume and mechanical properties was determined from 3D surface scans. Therefore, the optimal participant positioning and 3D scanner handling had to be defined in order to detect breast shape.

\section{Methods}

\subsection{Participants}

At the beginning of the project, 3 pilot study participants P1 to P3 (age 55 / 30 / 26 years, bra size 85E / 75AA / 75B) were recruited for repeatability tests of the 3D scanner. In the research project, 59 women participated. All participants signed informed consent, and the study was approved by the ethics committee of University of Vienna.

\subsection{Scanning positions}

As female upper body area implies special requirements for participant positioning, several positions proposed in literature were considered. In order to calculate mechanical properties of breast tissue, at least two positions putting the female breast under different gravity loading were necessary. For the upright view in sitting position, the abdominal region sometimes interfered with the breast region leading to a change in breast shape and occlusion of the lower breast. Therefore, standing position was used to overcome this problem. Primarily, a wooden plate with markers was considered as a back wall to ensure perfectly upright view. However, in order to calculate bust and underbust circumference, the participant must stand freely without any support of the back. As the difference in breast shape between freely standing and back supported upright view was rather low, the freely standing position on a turntable was used.

For the second position, either prone or supine would lead to different gravity loading $[15,17]$. Forward bent (prone) positions proved to be uncomfortable even for participants with average cup size due to the weight of the breast. Scanning was also quite difficult with the 3D scanner and lighting below the participant. Lying on the back (supine) was the most comfortable position both for the participant and the researcher, as the whole breast region was visible and artificial ceiling light was sufficient.

With the arms hanging down left and right to the body, the lateral region of the breast is partly covered. With the arms up as in [13], e.g. supported horizontally or on the head, the vertical position of nipple changes significantly. Therefore, the best compromise between visibility and natural shape of breast was a position with the arms supported at the hips similar as in [15]. The location of the hands was changed from the iliac crest with the thumbs pointing backwards to placing the whole palm loosely on the anterior superior iliac spine (see figure 1), for both upright and supine position. 




Fig. 1. Arm position with palms placed loosely on anterior superior iliac spine in upright position (P1).

\subsection{D scanner settings}

The $1^{\text {st }}$ generation Sense ${ }^{\text {TM }}$ (3D Systems Inc., Rock Hill, SC, USA) is a low-cost hand-held 3D scanner including free software Sense (V2.2) [1]. When starting Sense software, the user can decide between person and object. In case of person, the next distinction is between head or full body, in case of object between small, medium or large object. The only difference is the ob ject $\mathrm{Si}$ ze or scan volume, preset with an edge length of $0.4 \mathrm{~m}$ (small), $0.6 \mathrm{~m}$ (medium) or $2.0 \mathrm{~m}$ (large). For Custom, width, height and depth can be set individually between 0.2 and $3.0 \mathrm{~m}$. In case of female torso, we set about $1.0 \mathrm{~m}$ in all three dimensions according to the height and corpulence of the participant in upright position, whereas in supine position the depth can be reduced. Resolution was set to high, which is not directly associated with a numeric value. The scanner is limited to 400.000 triangles per scan, so it is assumed that the final resolution, i.e. the edge length of triangular mesh elements, is presumably calculated from scan volume and resolution settings. The chosen settings result in an element size of maximum $3 \mathrm{~mm}$ (see figure 2).



Fig. 2. Close-up view of mesh at breast region with element size of maximum $3 \mathrm{~mm}(P 1)$.

\subsection{Lighting}


The most important issue in 3D scanning is lighting. Not only the color texture mapping, but also the geometry of the scan suffers from inadequate lighting. The user guide [18] suggests that it is optimized for indoor use, so any window should be occluded avoiding direct sunlight. In order to reduce shadows, additional light with equal intensity over the whole subject being scanned might be needed. In case of artificial ceiling light, dark stripes occurred on the breast region which interfered with the markups (see figure 3). For the upright position, artificial ceiling light was turned off and the participant was lighted by a spotlight at about $45^{\circ}$ from above to avoid blinding. For the supine position, six LED array lamps mounted to the ceiling light were used.

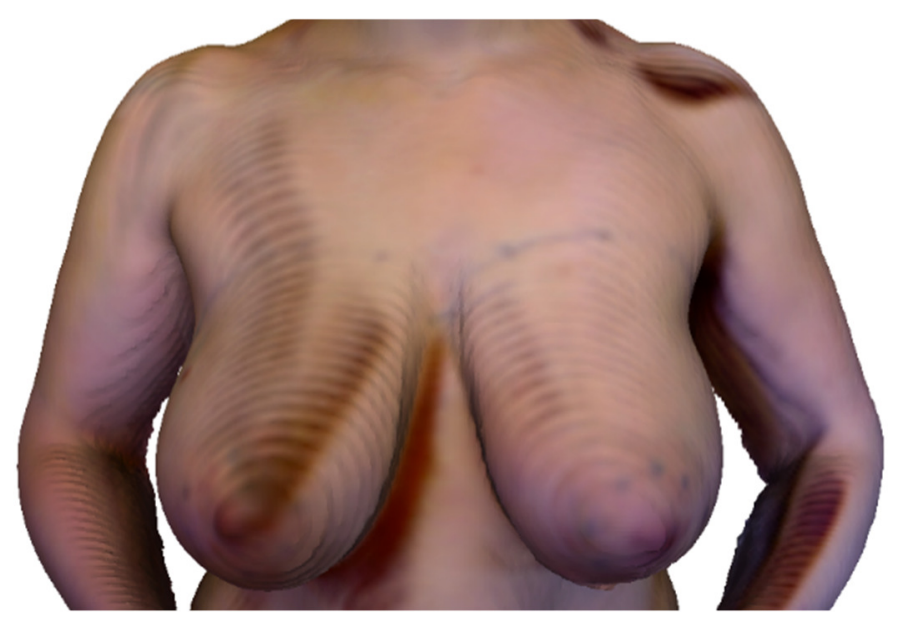

Fig. 3. Dark stripes caused by artificial ceiling light in upright position (P1).

\subsection{Scanning process}

For the upright position, the participant stepped on a turntable and placed her palms on the anterior superior iliac spine. The participant was advised to breathe in and out and then hold her breath or at least not to breathe deeply for the duration of scanning to keep her position unchanged. The scanner should be within a range of 40 to $150 \mathrm{~cm}$ from the participant, anything outside this range will not be recognized. Therefore, the scanner was mounted on a tripod about $1 \mathrm{~m}$ away from the participant. In preview mode, the whole participant should be visible on the screen and the target ring should be at the center of the participant [18]. When starting the scan, the first picture defines the coordinate system to which all other points are assigned. This first picture was taken in frontal view, then the turntable was turned by $45^{\circ}$ in one direction, once all around in the other direction, and finally remaining gaps were closed by moving the scanner around the participant. This procedure ensured optimal reproduction of breast region and avoided problems in closing the volume. Especially when passing the elbows, which are further away from the body, the front and back side sometimes couldn't be matched properly, resulting in "doubled shoulders".

One of the most frequent problems in scanning is losing tracking. This can occur if the participant gets outside the scan volume or due to large spatial changes during pausing the scan. The scanner has a maximal image throughput of $30 \mathrm{fps}$, so moving the scanner (or the participant on the turntable) too fast causes the same problem. If the scanner has lost tracking, the scanner (or the participant on the turntable) should be moved back to the last view before the error message, and the scanning process can be resumed.

\subsection{Data processing}

When the scanning process is finished, the Sense software automatically creates a model by removing points which are considered artefacts. If object recognition is turned off during the scan, more objects including the background are detected during the scanning process. Some of these additional objects are also removed during model creation. The user can further crop or erase the model and, if desired, solidify the model. Solidification means automatic gap closing, which can also be performed later in other software, such as MeshLab [2] or Blender [19]. In the last step, brightness and contrast can be adapted, and the Touch Up tool provides smoothing of the surface. The scans can be saved in *.obj, ${ }^{*}$.stl, *.ply or *.wrl format, whereby the binary *. stl saves only geometry; all other formats include color information. 
For anonymization of the scans, only the scan volume between chin and umbilicus was kept. If this was done by cropping or erasing in Sense software, a severe data issue was discovered. When re-opening the scan in any other software, only the selected faces were deleted, while the points including color information were still present. From these points, all information could still be retrieved. Effective anonymization therefore required an additional processing step. Either the scan was re-opened in Sense software and saved again (without any changes), or the loose points were deleted in any other software.

\subsection{Dimensional accuracy and repeatability}

Before using the Sense ${ }^{\mathrm{TM}} 3 \mathrm{D}$ scanner for research, dimensional accuracy has to be ensured. For this purpose, rigid plastic mannequins with markups, so-called fiducial points, are used $[4,15,20]$. The distances "incisura jugularis to umbilicus height" and "nipple distance" were measured on the real mannequin using manual anthropometry compasses. From the 3D scan, the same distances were measured using virtual measurement in MeshLab (v1.3.4BETA). The deviation between these two methods provided a measure for dimensional accuracy of the scanner.

Repeatability of scans was determined calculating average error parameter from 5 scans in upright and supine position using the alignment procedure in MeshLab. The first mesh is glued by Glue Here Mesh to fix its position. The second mesh is moved by Point Based Glueing using at least 4 matching pairs of fiducial points, which are manually picked on both meshes in consistent order (see figure 4). This procedure was repeated with 19 pairs of fiducial points to check whether the alignment was influenced by the choice of fiducial points. The average error, which is calculated after processing the alignment, takes into account all acquired points of the mannequin instead of single measurements as in [20].

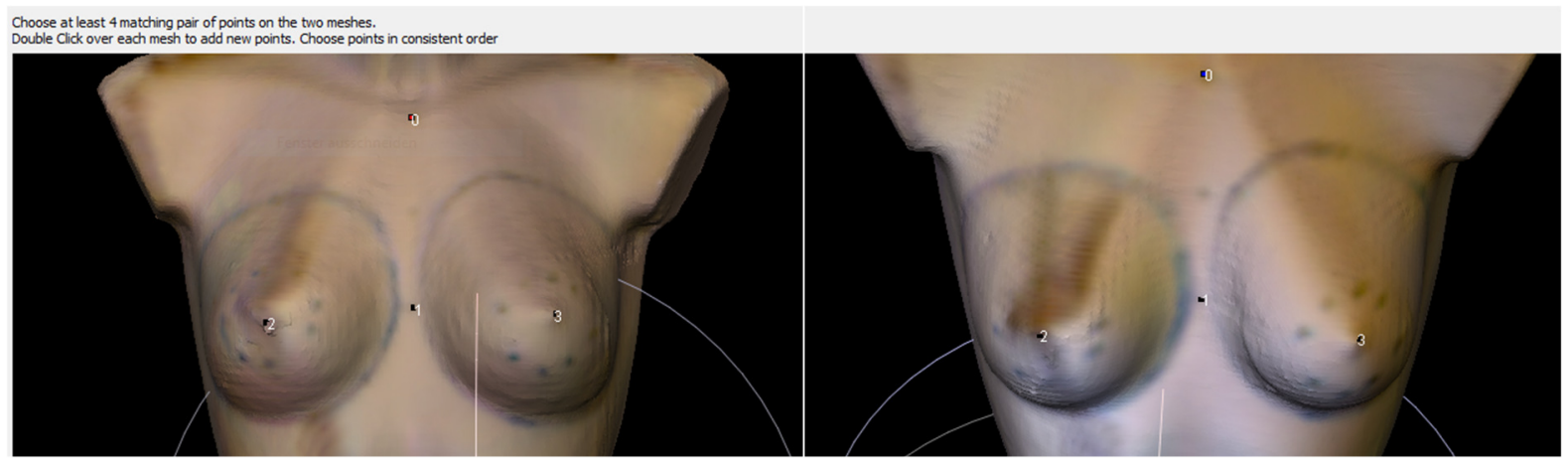

Fig. 4. Alignment procedure in MeshLab using 4 pairs of fiducial points.

As mannequins do not show relative displacements due to skin or soft tissue movements nor posture changes, similar repeatability measurements were carried out on 3 pilot study participants.

\section{Results and Discussion}

\subsection{Dimensional accuracy}

Table 1 shows the distances "incisura jugularis to umbilicus height" (see figure 5) and "nipple distance" measured manually on the mannequin and virtually from the 3D scan. The deviation was $0.1 \%$ and $-0.03 \%$, respectively. Considering the element size of maximum $3 \mathrm{~mm}$, this error could increase to maximum 1 to $2 \%$ when picking a neighboring point instead. The manufacturer information on resolution is given as spatial $\mathrm{x} / \mathrm{y}$ resolution of $0.9 \mathrm{~mm}$ and depth resolution of $1 \mathrm{~mm}$ for a scan volume with an edge length of $0.5 \mathrm{~m}$ [1], which is smaller but comparable to the scan volume in this use case. These values can be assumed to be reliable.

Table 1. Comparison of manual and virtual measurements of mannequin.

\begin{tabular}{|l|l|l|l|l|}
\hline \multirow{2}{*}{ Distance } & \multicolumn{2}{|l|}{ Measurement } & Deviation \\
\cline { 2 - 5 } & Manual & Virtual & Absolute & Relative \\
\hline incisura jugularis to umbilicus height & $335 \mathrm{~mm}$ & $335.34 \mathrm{~mm}$ & $0.34 \mathrm{~mm}$ & $0.1 \%$ \\
\hline nipple distance & $172 \mathrm{~mm}$ & $171.95 \mathrm{~mm}$ & $-0.05 \mathrm{~mm}$ & $-0.03 \%$ \\
\hline
\end{tabular}


Comparable values for dimensional accuracy could be found e.g. for the Minolta Vivid 910 scanner, where the mean value of the coefficient of variation for several dummy measurements was $3.5 \%$ [20]. Root mean square error between average acquired surface and CT scan of an anthropomorphic phantom (female torso) was $0.748 \mathrm{~mm}$ for the Minolta Vivid 910 scanner [21]. For the 3dMDtorso imaging system, the error between supine and upright measurements of a rigid mannequin was within $1 \%$ [15]. Global error values could be found only for mannequin head measurements using 3dMDhead system in the range of $0.2 \mathrm{~mm}$ [22], which is about $0.1 \%$ of head dimensions.



Fig. 5. Measuring incisura jugularis to umbilicus height of mannequin (335.34 mm) in MeshLab.

\subsection{Repeatability}

The mean average error when aligning 5 scans of the mannequin in upright and supine position was $0.433 \mathrm{~mm}$ and $0.256 \mathrm{~mm}$, respectively (see table 2), which indicates good accordance (see figure 6). Using 19 instead of 4 pairs of points for alignment did not affect this value. Keeping in mind that the scanner is optimized for human skin and not for plastic surface, which causes reflections especially in the upright position, this value can be judged very low.

Table 2. Average error for alignment of mannequin scans in MeshLab.

\begin{tabular}{|l|l|l|l|l|l|}
\hline \multirow{2}{*}{ Scanning position } & \multicolumn{5}{|l|}{ Average error to Scan_01 } \\
\cline { 2 - 6 } & Scan_02 & Scan_03 & Scan_04 & Scan_04 & Mean \\
\hline upright & $0.401 \mathrm{~mm}$ & $0.396 \mathrm{~mm}$ & $0.501 \mathrm{~mm}$ & $0.433 \mathrm{~mm}$ & $0.433 \mathrm{~mm}$ \\
\hline supine & $0.289 \mathrm{~mm}$ & $0.256 \mathrm{~mm}$ & $0.247 \mathrm{~mm}$ & $0.232 \mathrm{~mm}$ & $0.256 \mathrm{~mm}$ \\
\hline
\end{tabular}

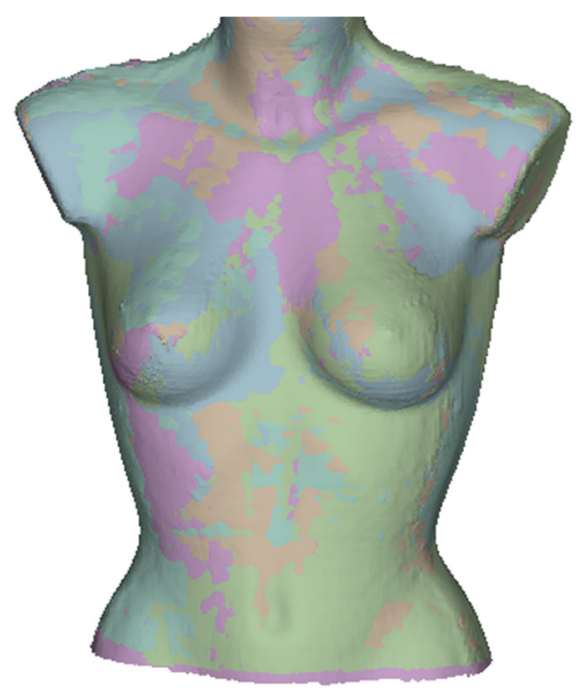

Fig. 6. Alignment of 5 upright scans of the mannequin in MeshLab. 
The repeatability measurements for 3 pilot study participants showed a mean average error of 1.395 $\mathrm{mm}$ (upright) and $1.271 \mathrm{~mm}$ (supine) (see table 3). The mean average error was slightly lower for the supine position and highest for P1 with the largest cup size (85E). Compared to the element size of maximum $3 \mathrm{~mm}$ and keeping in mind that human position can't exactly be reproduced, this error is acceptable.

Table 3. Average error for alignment of participant scans in MeshLab.

\begin{tabular}{|l|l|l|l|l|}
\hline \multirow{2}{*}{ Scanning position } & \multicolumn{3}{|l|}{ Mean average error to Scan_01 } & \\
\cline { 2 - 5 } & P1 & P2 & P3 & Mean \\
\hline upright & $1.481 \mathrm{~mm}$ & $1.355 \mathrm{~mm}$ & $1.347 \mathrm{~mm}$ & $1.395 \mathrm{~mm}$ \\
\hline supine & $1.596 \mathrm{~mm}$ & $1.323 \mathrm{~mm}$ & $0.923 \mathrm{~mm}$ & $1.271 \mathrm{~mm}$ \\
\hline
\end{tabular}

Repeatability was verified e.g. for the Minolta Vivid 910 scanner, using both dummy and test persons [20]. Mean of scanner repeatability among 3 female torso phantom acquisitions was $0.406 \mathrm{~mm}$ for this scanner, for participant data this value was between 1.239 and $1.878 \mathrm{~mm}$ [21].

\section{Conclusions}

The $1^{\text {st }}$ generation Sense ${ }^{\mathrm{TM}}$ does not require nor enable calibration, so the user has to rely on the accuracy of its auto-calibration. The advantages of this scanner can be summarized as follows: It is cheap compared to other commercial 3D scanners, lightweight and handy and the included free software Sense (V2.2) is intuitive and easy-to-use. It provides quite good scan quality if lighting conditions are adequate.

On the other hand, a lot of limiting issues have been identified: The familiarization with the scanner handling and the development of the final scanning procedure takes several hours and it has to be repeated for different size and shape of objects as well as lighting conditions. The scanner seems to save the current lighting conditions, which sometimes requires a test scan when changing lighting between scans. Sharp edges are not reproduced properly and appear rounded, just like occluded concave areas e.g. the inframammary fold. The scanner is delivered with a USB cable with a cord length of $2 \mathrm{~m}$. Extension cables reduces data transmission speed, so a maximum of $1 \mathrm{~m}$ extension is acceptable due to our experience. When scanning larger objects or when moving around the participant, this maximum distance has to be considered. Instead of moving the scanner, the use of turntables has become established for objects as well as humans. Placing the participant on a turntable keeps the required distance between scanner and object relatively constant, thus reducing scanning time and facilitating the participant to keep the same position. The scanner should be placed on a tripod for the first picture using a level to ensure the vertical axis to be precise. Data processing during model creation automatically removes point which have previously been detected during the scanning process and the software considers as artefacts. This automatic deletion sometimes also erased points which actually belong to the model, and it concerns arbitrary areas of the female torso.

To our knowledge, only Koban, Schenck and Giunta [3] have published experiences with this scanner so far, judging it capable of capturing breast surfaces. The relatively low mean average error in repeatability allowed for capturing only one scan of each participant and position, reducing time demand in scanning and data processing. Compared to 3D scanning systems which require stitching and merging of several scans from different angles [23], the Sense ${ }^{\mathrm{TM}}$ scanner requires only one scan. Considering the good dimensional accuracy and repeatability, the low-cost Sense ${ }^{\mathrm{TM}}$ scanner can be used in research projects dealing with human body geometric measurements. In addition to the $1^{\text {st }}$ generation Sense ${ }^{\mathrm{TM}}$ scanner operating on Windows 7 used in this research project, iSense ${ }^{\mathrm{TM}}$ scanner mounted to iPad and $2^{\text {nd }}$ generation Sense ${ }^{\mathrm{TM}}$ scanner operating on Windows 8 or 10 (together with updated software Sense V3.0) are available.

\section{Acknowledgments}

This work is part of the research project "Sports bra optimization by Finite Element simulation of interaction between textile and female breast tissue (FEM-Sports-Bra)" (January 2016 to February 2018), funded by Vienna Business Agency. 


\section{References}

[1] Sense ${ }^{T M} 3 D$ scanner, https://www.3dsystems.com/shop/sense, accessed 2018.

[2] P. Cignoni, M. Callieri, M. Corsini, M. Dellepiane, F. Ganovelli and G. Ranzuglia, "MeshLab: an Open-Source Mesh Processing Tool", in $6^{\text {th }}$ Eurographics Italian Chapter Conference, Salerno, Italy, 2008, pp.129-136.

[3] K. C. Koban, T. L. Schenck and R. E. Giunta, "Using Mobile 3D Scanning Systems for Objective Evaluation of Form, Volume, and Symmetry in Plastic Surgery: Intraoperative Scanning and Lymphedema Assessment", in Proc. of $7^{\text {th }}$ Int. Conf. on 3D Body Scanning Technologies, Lugano, Switzerland, 2016, pp.130-131, http://dx.doi.org/10.15221/16.130.

[4] S. B. Choppin, H. Probst, A. Goyal, S. Clarkson and J. Wheat, "Breast Volume Calculation Using a Low-Cost Scanning System", in Proc. of $4^{\text {th }}$ Int. Conf. on 3D Body Scanning Technologies, Long Beach, USA, 2013, pp.12-20, http://dx.doi.org/10.15221/13.012.

[5] L. Zhao, G. P. Reece, M. C. Fingeret and F. Merchant, "Multi-View 3D Data Fusion for Visualization of the Inframammary Fold in Women with Ptotic Breasts", in Proc. of $5^{\text {th }}$ Int. Conf. on 3D Body Scanning Technologies, Lugano, Switzerland, 2014, pp.29-38, http://dx.doi.org/10.15221/14.029.

[6] K. E. Peyer, M. Morris and W. I. Sellers, "Subject-specific body segment parameter estimation using 3D photogrammetry with multiple cameras", in PeerJ, Vol.3, 2015, e831, https://doi.org/10.7717/peeri.831.

[7] B. Bonnechère et al., "Determination of the precision and accuracy of morphological measurements using the Kinect ${ }^{\mathrm{TM}}$ sensor: comparison with standard stereophotogrammetry", in Ergonomics, Vol.57, No.4, 2014, pp.622-631, http://dx.doi.org/10.1080/00140139.2014.884246.

[8] 3D Scanner Series. Capture your life in 3D today for tomorrow's digital world, https://www.xyzprinting.com/en-GB/product-level/PROFESSIONAL/3d-scanner-series, accessed 2018.

[9] EinScan-Pro+. Professional High-performance Handheld 3D Scanner, https://www.einscan.com/einscan-pro-plus, accessed 2018.

[10] Artec Eva. Fast 3D scanner for professionals, https://www.artec3d.com/portable-3d-scanners/artec-eva, accessed 2018.

[11] H. P. Oliveira, P. Patete, G. Baroni and J. S. Cardoso, "Development of a BCCT Quantitative 3D Evaluation System through Low-Cost Solutions", in Proc. of $2^{\text {nd }}$ Int. Conf. on 3D Body Scanning Technologies, Lugano, Switzerland, 2011, pp.16-27, http://dx.doi.org/10.15221/11.016.

[12] B. Shin, R. Venkatramani, P. Borker, A. Olch, J. Grimm and K. Wong, "Spatial Accuracy of a Low Cost High Resolution 3D Surface Imaging Device for Medical Applications", in International Journal of Medical Physics, Clinical Engineering and Radiation Oncology, Vol.2, 2013, pp.45-51, http://dx.doi.org/10.4236/ijmpcero.2013.22007.

[13] H. P. Oliveira, J. S. Cardoso, A. Magalhães and M. J. Cardoso, "Methods for the Aesthetic Evaluation of Breast Cancer Conservation Treatment: A Technological Review", in Current Medical Imaging Reviews, Vol.9, 2013, pp.32-46, http://dx.doi.org/10.2174/1573405611309010006.

[14] C. E. Coltman, D. E. McGhee and J. R. Steele, "Three-dimensional scanning in women with large, ptotic breasts: implications for bra cup sizing and design", in Ergonomics, Vol.60, No.3, 2017, pp.439-445, http://dx.doi.org/10.1080/00140139.2016.1176258.

[15] G. P. Reece et al., "3D surface imaging of the human female torso in upright to supine positions", in Medical Engineering \& Physics, Vol.37, No.4, 2015, pp.375-383, http://dx.doi.org/10.1016/j.medengphy.2015.01.011.

[16] Y. Liu, C. L. Istook, K. Liu and J. Wang, "Innovative method for creating fitted brassiere wire prototype based on transformation matrix algorithm", in The Journal of the Textile Institute, Vol.7, 2017, pp.1-6, http://dx.doi.org/10.1080/00405000.2017.1326366.

[17] A. P. del Palomar, B. Calvo, J. Herrero, J. López and M. Doblaré, "A finite element model to accurately predict real deformations of the breast", in Medical Engineering \& Physics, Vol.30, No.9, 2008, pp.1089-1097, https://doi.org/10.1016/j.medengphy.2008.01.005.

[18] Sense TM2 3D scanner. Capture your world in 3D. User Guide. Original Instructions, http://cubify.s3.amazonaws.com/Scanners/Sense/sense 2nd gen user guide win.pdf, accessed 2018. 
[19] Open Source 3D creation. Free to use for any purpose, forever, https://www.blender.org/,accessed 2018.

[20] M. Eder, F. Armbrecht, S. Raith, A. Volf, A. Zimmermann, N. A. Papadopulos and L. Kovacs, "Optimisation of Three-Dimensional Imaging of the Breast Region with 3-D Laser Scanners", in Proc. of Int. Conf. on 3D Body Scanning Technologies, Lugano, Switzerland, 2010, pp.87-88, http://dx.doi.org/10.15221/10.082.

[21] P. Patete, M. Eder, S. Raith, A. Volf, L. Kovacs and G. Baroni, "Comparative Assessment of 3D Surface Scanning Systems in Breast Plastic and Reconstructive Surgery", in Surgical Innovation, Vol.20, No.5, 2012, pp.509-515, http://dx.doi.org/10.1177/1553350612463443.

[22] C.-H. J. Tzou, N. M. Artner, I. Pona, A. Hold, E. Placheta, W. G. Kropatsch and M. Frey, "Comparison of three-dimensional surface-imaging systems", in Journal of Plastic, Reconstructive \& Aesthetic Surgery, Vol.67, No.4, 2014, pp.489-497, http://dx.doi.org/10.1016/j.bjps.2014.01.003.

[23] S. Raith, Applications of Computational Biomechanics in Reconstructive Cranio-Maxillofacial and Plastic Surgery, Technische Hochschule Aachen, Germany, 2014. 